University of Nebraska - Lincoln

DigitalCommons@University of Nebraska - Lincoln

2-15-1970

\title{
Existence of Fixed Poles and Their Role in Conspiracy
}

\author{
Paul Finkler \\ University of Nebraska-Lincoln, pfinkler1@unl.edu
}

Follow this and additional works at: https://digitalcommons.unl.edu/physicsfinkler

Part of the Physics Commons

Finkler, Paul, "Existence of Fixed Poles and Their Role in Conspiracy" (1970). Paul Finkler Papers. 5. https://digitalcommons.unl.edu/physicsfinkler/5

This Article is brought to you for free and open access by the Research Papers in Physics and Astronomy at DigitalCommons@University of Nebraska - Lincoln. It has been accepted for inclusion in Paul Finkler Papers by an authorized administrator of DigitalCommons@University of Nebraska - Lincoln. 


\title{
Existence of Fixed Poles and Their Role in Conspiracy
}

\author{
PAUL Finkler \\ Behlen Laboratory of Physics, University of Nebraska, Lincoln, Nebraska 68508
}

(Received 28 August 1969)

\begin{abstract}
It is shown that unitarity allows fixed poles at certain nonsense points of either right or wrong signature. The conditions for the existence of these poles are found. These conditions are then used to locate the poles allowed in hadronic reactions. Possible mechanisms for the poles are considered. It is then argued that fixed poles provide the most natural explanation of the conspiracy phenomenon.
\end{abstract}

\section{INTRODUCTION}

$\mathrm{T}^{\mathrm{H}}$ HE existence of conspiracies ${ }^{1}$ and the apparent need for Regge cuts ${ }^{2}$ are two of the more vexing aspects of the Regge-pole theory of high-energy scattering. Conspiracy is now firmly established as the only way to explain the sharp forward peaks in charged-pion photoproduction and $n-p$ charge-exchange scattering. ${ }^{3}$ The explanation requires the existence of terms with the same energy dependence at $t=0$ in amplitudes of opposite parity. There is no apparent dynamical reason, however, for the coincidence of opposite-parity $t$ channel trajectories at $t=0$. One proposed resolution of this difficulty involves the use of Regge cuts. ${ }^{4}$ Moving cuts appear to be necessary to prevent the development of essential singularities, via the Gribov-Pomeranchuk phenomenon, ${ }^{5}$ in relativistic scattering amplitudes of definite signature. Such cuts as are used in high-energy phenomenology have properties similar to what one finds from calculating the simultaneous exchange of two or more Regge trajectories. Thus the cuts are not necessarily associated with a definite parity and their contributions can presumably conspire. The arguments of Mandelstam ${ }^{2}$ indicate, however, that the cuts needed to prevent the essential singularities from developing arise from the third double spectral function $\left(\rho_{s u}\right.$ for cuts in the continued $t$-channel partial-wave amplitudes). Here, it is difficult to see why contributions from the third double-spectral function, or the manifestly nonperipheral diagrams associated with it (see Fig. 1), should dominate some processes at high energies near $t=0$.

From a practical point of view also, conspiracies and cuts pose problems. For example, one must determine if trajectories associated with physical particles actually conspire and, if so, into which conspiracy class ${ }^{6}$ these trajectories fall. Most of the known particles appear to be ordinary but some, including the pion, are thought to

${ }^{1}$ D. V. Volkov and V. N. Gribov, Zh. Eksperim. i Teor. Fiz. 44, 1068 (1963) [English transl.: Soviet Phys.-JETP 17, 720 (1963)].

${ }^{2}$ S. Mandelstam, Nuovo Cimento 30, 1148 (1963); S. Mandelstam and L. L. Wang, Phys. Rev. 160, 1490 (1967).

${ }^{3} \mathrm{~L}$. Bertocchi, in Proceedings of the International Conference on Elementary Particles, Heidelberg, 1967, edited by H. Filthuth (North-Holland Publishing Co., Amsterdam, 1968)

${ }^{4}$ J. Fr $\phi y l a n d$ and D. Gordon, Phys. Rev. 177, 2500 (1969).

${ }^{5} \mathrm{~V}$. N. Gribov and I. Ya. Pomeranchuk, Phys. Letters 2, 232 (1962).

${ }^{6}$ M. Toller, Nuovo Cimento 37, 631 (1965); 53 A, 671 (1967).

be extraordinary. If cuts are important at high energy, in the context of conspiracy or otherwise, then the ultimate goal of performing dynamical calculations of the Regge parameters would be much more difficult to attain. This is due to the continuum of parameters associated with the cuts. Of more imminent concern, of course, is that cuts complicate the phenomenological determination of the Regge parameters.

Clearly, cuts and conspiring trajectories make the Regge theory somewhat unwieldy. The theory would be

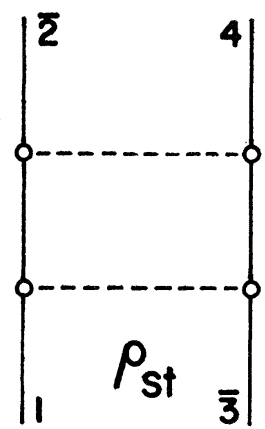

(a) (b)

.

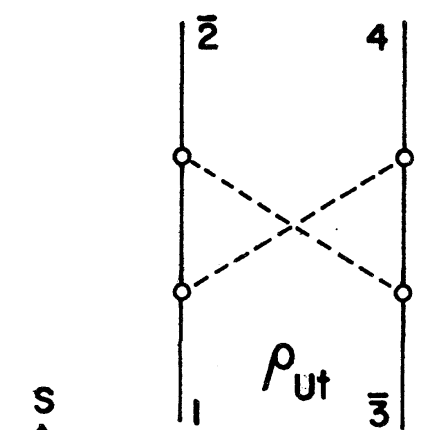

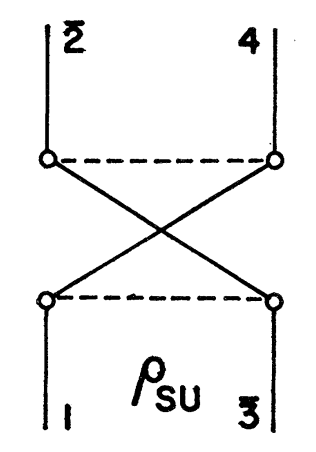

(c)

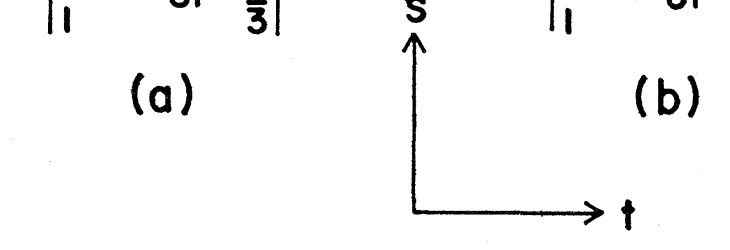

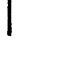

Fig. 1. Simplest Feynman diagrams associated with the (a) first, (b) second, and (c) third double-spectral function, and (d) the simplest diagram of the set which leads to Mandelstam cuts in the $J$ plane of the $t$ channel. 
made more tractable if a simpler mechanism were allowed which could explain the conspiracy phenomena and/or account for those problems which appear to necessitate cuts. In this paper we revive the old idea ${ }^{7}$ that fixed $J$-plane poles, not associated with the third double-spectral function, exist in certain amplitudes and contribute to the asymptotic behavior of the scattering amplitude. It is a common belief that such fixed poles violate unitarity and this remains true under certain conditions. In Sec. II we demonstrate the breakdown of the no-fixed-pole requirement and deduce the conditions under which fixed poles are allowed. In Sec. III we locate the fixed poles allowed in hadronic amplitudes and in photoproduction. An argument against conspiring trajectories and for conspiring fixed poles is presented in Secs. IV and V, and our conclusions are summarized in the final section.

\section{BREAKDOWN OF NO-FIXED- POLES RULE}

In this section it will be shown that unitarity does not always prohibit fixed poles at nonsense points of right or wrong signature. The discussion is purposely limited to nonsense points because for these points it is unnecessary to invoke $a$ priori a dynamical mechanism for the poles; the necessary singularities are explicitly contained in the definition of the continued partial-wave amplitudes. A dynamical mechanism must be found, of course, for a pole actually to exist, but the mechanism can be sought a posteriori.

Another limitation to be made is that transitions between states of odd baryon number will not be considered. In this way the complications of the generalized MacDowell symmetry will be avoided. ${ }^{8}$ Recall that it is this symmetry which leads automatically to conspiracy between opposite-parity fermion trajectories. This probably unnecessary limitation also permits the use of the appropriate Mandelstam variable instead of the total energy.

A brief outline of the following material is in order. First, the relevant properties of helicity amplitudes and the continued amplitudes are reviewed. Then it is shown, by means of a simple example, why fixed poles are sometimes forbidden and sometimes allowed. The particular example used displays all of the ingredients needed for the general demonstration. For this reason, the general results are then stated without proof. An

\footnotetext{
${ }^{7}$ The idea that right-signature fixed poles are possible has occurred to several authors but, for some reason, the idea was never followed up. Indeed, the same authors go on to ignore or reject the existence of these poles. See, for example, a reference to D. Amati by W. Drechsler, Nuovo Cimento 53 A, 115 (1968) also the comments by P. D. B. Collins and E. J. Squires, in Springer Tracts in Modern Physics, edited by G. Hohler (SpringerVerlag, Berlin, 1968), Vol. 45, pp. 132-133; and R. G. Newton, The Complex j-Plane (W. A. Benjamin, Inc., New York, 1964), Footnote 8, Chap. 16.

${ }^{8}$ S. MacDowell, Phys. Rev. 116, 774 (1959); Y. Hara, ibid. 136, 507 (1964)
}

outline of the essential points is given, however, wherever it is needed.

\section{A. Kinematic Preliminaries}

At high energies $(s \rightarrow \infty)$ and near the forward direction $(t \approx 0)$, the $s$-channel reaction $1+\overline{3} \rightarrow \overline{2}+4$ is governed by the rightmost $J$-plane singularities of the amplitudes for the $t$-channel reaction $1+2 \rightarrow 3+4$. The helicity amplitudes for the last reaction have the partial-wave expansion ${ }^{9}$

$$
\phi_{\lambda_{3} \lambda_{4}, \lambda_{1} \lambda_{2}}(t, z)=\sum_{J=M}^{\infty}(2 J+1) \phi^{J}{ }_{\lambda_{3} \lambda_{4}, \lambda_{1} \lambda_{2}}(t) d_{\lambda \mu}{ }^{J}(\theta),
$$

where the helicity $\lambda_{i}$ for particle $i$ with spin $\sigma_{i}$ can take on values in integral steps from $-\sigma_{i}$ to $+\sigma_{i}$, and where $\lambda=\lambda_{1}-\lambda_{2}, \mu=\lambda_{3}-\lambda_{4}, M=\max (|\lambda|,|\mu|)$, and $z=\cos \theta{ }^{10}$

The unitarity condition ${ }^{11}$ for the partial-wave helicity amplitudes $\phi^{J}{ }_{\lambda_{3} \lambda_{4}, \lambda_{1} \lambda_{2}}$ is given by

$$
\begin{aligned}
\phi^{J}{ }_{\lambda_{3} \lambda_{4}, \lambda_{1} \lambda_{2}}(t)- & \phi^{J}{ }_{\lambda_{1} \lambda_{2}, \lambda_{3} \lambda_{4}}(t)^{*} \\
& =2 i \sum_{(5+6)} \phi^{J}{ }_{\lambda_{3} \lambda_{4}, \lambda_{5} \lambda_{6}}(t) \phi^{J}{ }_{\lambda_{1} \lambda_{2}, \lambda_{5} \lambda_{6}}(t)^{*},
\end{aligned}
$$

where $\lambda_{5}$ and $\lambda_{6}$ are the helicities of the two, possibly "composite," particles in the $t$-channel state $5+6$. The sum extends over all such states with the allowed quantum numbers and with threshold $t_{56}<t$.

At this point it is convenient to make the simplifying but, for our purposes, inessential assumptions of parity and time-reversal invariance; these assumptions imply, respectively, that amplitudes with opposite sets of helicity labels are proportional and that amplitudes with initial and final states reversed are equal. We are then free to work with the set of independent amplitudes with $|\mu| \leq \lambda=M$.

The helicity amplitudes $\phi_{\lambda_{3} \lambda_{4}, \lambda_{1} \lambda_{2}}$ have kinematic singularities in the variables $t$ and $z$. If the "half-angle factors"

$$
\xi_{\lambda \mu}(z)=(1-z)^{|\lambda-\mu| / 2}(1+z)^{|\lambda+\mu| / 2}
$$

are extracted, however, then the amplitudes

$$
\bar{\phi}_{\lambda_{3} \lambda_{4}, \lambda_{1} \lambda_{2}}(t, z)=\phi_{\lambda_{3} \lambda_{4}, \lambda_{1} \lambda_{2}}(t, z) / \xi_{\lambda \mu}(z),
$$

and their parity-conserving counterparts

$$
\bar{\phi}_{\lambda \mu} P=\frac{1}{2}\left(\bar{\phi}_{\lambda_{3} \lambda_{4}, \lambda_{1} \lambda_{2}}+P \bar{\phi}_{-\lambda_{3}-\lambda_{4}, \lambda_{1} \lambda_{2}}\right),
$$

where $P= \pm 1$ are free of kinematic singularities in $z$. If it is now assumed that $\phi_{ \pm \lambda_{3} \pm \lambda_{4}, \lambda_{1} \lambda_{2}}$ is bounded by a polynomial of order $N$ in $z$, then $\bar{\phi}_{\lambda \mu}{ }^{P}$ satisfies the $N-\lambda$ times subtracted version of the fixed- $t$ dispersion

${ }^{9}$ M. Jacob and G. C. Wick, Ann. Phys. (N. Y.) 7, 404 (1959).

10 Properties of the rotation functions of the first kind, $d_{\lambda \mu}{ }^{J}$ and second kind, $e_{\lambda \mu}{ }^{J}$, may be found in W. Drechsler, Nuovo Cimento 53 A, 115 (1968).

11 The unitarity condition also serves to fix our normalization of $\phi^{J}(t)$. 
relation

$$
\bar{\phi}_{\lambda \mu}{ }^{P}(t, z)=\frac{1}{\pi} \int_{z 0}^{\infty} d z^{\prime}\left(\frac{\bar{\phi}_{\lambda \mu}{ }^{s}\left(t, z^{\prime}\right)}{z^{\prime}-z}+\frac{\bar{\phi}_{\lambda \mu}{ }^{u}\left(t,-z^{\prime}\right)}{z^{\prime}+z}\right),
$$

where $\bar{\phi}_{\lambda \mu}{ }^{s}(t, z)$ and $\bar{\phi}_{\lambda \mu}{ }^{u}(t, z)$ are the absorptive parts of $\bar{\phi}_{\lambda \mu}{ }^{P}(t, z)$ in the $s$ and $u$ channels, respectively. The integration is understood to extend as far down toward zero as is necessary to include all dynamical singularities of $\bar{\phi}_{\lambda \mu}^{P}$ in $z$.

It is now possible to define the continued partial-wave amplitudes of definite signature $\phi_{\lambda_{\mu}} P(J, t)_{S}$, where $S= \pm 1$ is the signature, by means of the FroissartGribov projection ${ }^{10}$

$$
\begin{aligned}
\phi_{\lambda \mu}{ }^{P}(J, t)_{S}= & \frac{1}{\pi} \int_{z 0}^{\infty} d z\left[\bar{\phi}_{\lambda \mu}{ }^{s}(t, z) \xi_{\lambda \mu}(z) e_{\lambda \mu}{ }^{J}(z)\right. \\
& \left.+S(-1)^{\lambda} \bar{\phi}_{\lambda \mu}{ }^{u}(t,-z) \xi_{\lambda,-\mu}(z) e_{\lambda,-\mu} J(z)\right] .
\end{aligned}
$$

This projection represents the unique $J$-plane continuation of the partial-wave amplitudes $\phi_{\lambda \mu}{ }^{J P}$ into the region $\operatorname{Re} J \geq N$, where the defining integral exists. In this region one can make the identification $\phi_{\lambda_{\mu}}{ }^{P}(J, t)_{S}$ $\equiv \phi_{\lambda \mu}{ }^{J P}(t)$ for integral $J$ with $S(-1)^{J}=1$. Hereafter, we consider the signature to be specified and drop the label $S$.

In order to obtain partial-wave amplitudes which are real-analytic in $J$ and $t$, it is necessary to extract from $\phi_{\lambda \mu}{ }^{P}(J, t)$ its remaining kinematic singularities in $t$. These singularities occur in two pieces: (1) the kinematic singularities and zeros of the full amplitude which depend on the spins and parities of the external particles (and the parity of the amplitude), and (2) the $J$-dependent kinematic zeros at the normal thresholds and pseudothresholds which reflect the different threshold behaviors of the various partial waves.

The importance of the kinematic factors, for our purposes, is that the continued partial-wave amplitude with these singularities removed is real-analytic in the cut $t$ and $J$ planes. The prescription for extracting these singularities is well known. ${ }^{3}$ In order to avoid repeating the lengthy formulas here, we simply attribute to $\phi_{\lambda_{\mu}}{ }^{P}(J, t)$ the real-analyticity property

$$
\phi_{\lambda \mu}{ }^{P}(J, t)=\phi_{\lambda \mu}{ }^{P}\left(J^{*}, t^{*}\right)^{*},
$$

keeping in mind that this equation is strictly true for the kinematic-singularity-free continued amplitude. What allows us to make this simplification without changing our results is that the kinematic factors have the form $c_{1}(t) c_{2}(t)^{J}$ and are thus analytic everywhere in the finite part of the $J$ plane.

The $J$-plane singularities of $\phi_{\lambda \mu}{ }^{P}(J, t)$ can arise from two sources: (1) from the asymptotic behavior of the absorptive parts of $\phi_{\lambda \mu}{ }^{P}(t, z)$ that generates the usual moving poles, and (2) from the fixed singularities of the functions $e_{\lambda \mu}{ }^{J}(z) .^{12}$ We will assume that the moving

${ }^{12}$ Fixed poles at nonsense points of right or wrong signature do not contribute to the asymptotic behavior of the absorptive parts of $\phi_{\lambda \mu} P(t, z)$. poles either are sufficiently far to the left or are isolated from the fixed singularities so that these poles (or cuts) can be explicitly separated off in the right-hand side of Eq. (4). ${ }^{13}$ We can now examine the fixed singularities appearing in $e_{\lambda \mu}{ }^{J}$. These singularities are of two types. ${ }^{3}$ First, if $\lambda>|\mu|$, there are square-root singularities $\left(J-J_{0}\right)^{-1 / 2}$ at the nonsense-sense (n-s) points $J_{0}=\lambda-1, \lambda-2, \ldots,|\mu|$ and $-|\mu|-1,-|\mu|-2, \ldots$, $-\lambda$. Then there are poles $\left(J-J_{0}\right)^{-1}$ at the nonsensenonsense (n-n) points $|\mu|-1,|\mu|-2, \ldots,-|\mu|$ and the points $-\lambda-1,-\lambda-2,-\lambda-3, \ldots$

We now adopt the convention that the cuts are drawn in a nested fashion from a given n-s point to its reflection about $J=-\frac{1}{2}$, a cut in the interior of the nest appears on all sheets of the outer cuts. Similarly, a pole at an interior n-n point appears on all sheets of the outer cuts. This convention for the cuts is equivalent to any other we might choose, but it makes the arguments presented below somewhat more transparent. The values of $J$ corresponding to the outermost cut will be called the SN interval for the amplitude.

Near a singular point $J_{0}$ we will make the nature of the singularity explicit by writing

$$
\begin{aligned}
\phi_{\lambda \mu}(J, t) \underset{J \rightarrow J_{0}}{\longrightarrow} & \left\{\begin{array}{l}
\beta_{\lambda \mu}{ }^{n n}\left(J_{0 \pm}, t\right)\left(J-J_{0 \pm}\right)^{-1}+\gamma_{\lambda \mu}{ }^{n n}\left(J_{0 \pm}, t\right) \\
\beta_{\lambda \mu}{ }^{n s}\left(J_{0 \pm}, t\right)\left(J-J_{0 \pm}\right)^{-1 / 2}+\gamma_{\lambda \mu}{ }^{n s}\left(J_{0 \pm}, t\right)\left(J-J_{0 \pm}\right)^{1 / 2} \\
\beta_{\lambda \mu}{ }^{s s}\left(J_{0}, t\right)
\end{array}\right\}
\end{aligned}
$$

for n-n, n-s, and s-s points, respectively. The distinction between $J_{0+}$ and $J_{0-}$ occurs if the point is interior to an $\mathrm{SN}$ interval. At an $\mathrm{n}$-s point at the edge of the SN interval, or at an n-n point not in an SN interval, the distinction disappears; in this case the real-analyticity condition Eq. (5) leads to the relation

$$
\beta_{\lambda \mu}\left(J_{0}, t_{-}\right)=\beta_{\lambda \mu}{ }^{*}\left(J_{0}, t_{+}\right) \text {. }
$$

It is possible, of course, to factor out from the amplitude the square-root singularities. The existence or nonexistence of fixed poles, however, depends on the nonlinear unitarity condition. Consequently, these singularities will appear in the unitarity condition whether or not the amplitude is so modified.

The unitarity condition, i.e., the continuation of Eq. (2) into the $t$ and $J$ planes, is given by

$$
\phi_{\lambda \mu}\left(J, t_{+}\right)-\phi_{\lambda \mu}\left(J, t_{-}\right)=2 i \sum_{\nu} \phi_{\lambda \nu}\left(J, t_{+}\right) \phi_{\nu \mu}\left(J, t_{-}\right)
$$

or

$$
\begin{aligned}
\phi_{\lambda \mu}\left(J, t_{+}\right)-\phi_{\lambda \mu}\left(J^{*}, t_{+}\right)^{*} & =2 i \sum_{\nu} \phi_{\lambda \nu}\left(J, t_{+}\right) \phi_{\nu \mu}\left(J^{*}, t_{+}\right)^{*} .
\end{aligned}
$$

${ }^{13}$ Essentially the same assumption is made in the argument that the physical amplitudes $\phi^{J P}(t)$ agree with the continued amplitude $\phi^{P}(J, t)_{S}$ at right-signature sense values of $J$ whenever $M \leq J<N$. 
The last form follows from the condition of real analyticity, Eq. (5). The sums in these equations are still understood to extend over different states whenever necessary.

\section{B. Simple Example}

In order to show that fixed poles are possible, we consider for definiteness the elastic scattering of two particles of spin 0 and 2. We now make the critical assumption that is commonly thought to prohibit fixed poles, namely, that the elastic threshold for these two particles is the lowest threshold so that elastic unitarity holds for some range of $t$.

The nine independent helicity amplitudes $\phi_{\lambda \mu}{ }^{P}(J, t)$ for each signature are $\phi_{00}, \phi_{10}, \phi_{20}, \phi_{11}^{ \pm}, \phi_{21}^{ \pm}$, and $\phi_{22}{ }^{ \pm}$. Four of these amplitudes have an SN interval: For $\phi_{20}$ and $\phi_{21}^{ \pm}$the interval is $[1,-2]$, while for $\phi_{10}$ it is $[0,-1]$. Furthermore, $\phi_{20}$ has an additional cut from 0 to -1 .

Now we examine the point $J=1$, which is an s-s point for $\phi_{00}, \phi_{10}$, and $\phi_{11}^{ \pm}$, an n-s point at the edge of the SN interval for $\phi_{20}$ and $\phi_{21}^{ \pm}$, and an n-n point for $\phi_{22} \pm$. For none of these amplitudes is there a distinction between $J_{0_{+}}$and $J_{0_{-}}$in $\beta_{\lambda \mu}$. The unitarity condition for the amplitude with the $n-n$ point reads ${ }^{14}$

$$
\begin{aligned}
(J-1)^{-1}\left[\beta_{22} \pm(1, t)-\beta_{22} \pm(1, t)^{*}\right] & =2 i\left[(J-1)^{-1}\right. \\
\times\left\{\left|\beta_{20}(1, t)\right|^{2}+\left|\beta_{21}(1, t)\right|^{2}+\right. & \left.\left(\beta_{22^{ \pm}} \gamma_{22^{ \pm *}}+\beta_{22}{ }^{ \pm *} \gamma_{22}{ }^{*}\right)\right\} \\
& \left.+(J-1)^{-2}\left|\beta_{22}{ }^{+}(1, t)\right|^{2}\right] .
\end{aligned}
$$

The coefficient of $(J-1)^{-2}$ must vanish, which implies that $\beta_{22} \pm(1, t)=0$. The remaining terms are of order $(J-1)^{-1}$. A similar analysis of the other unitarity conditions also shows that

$$
\beta_{11}^{ \pm}(1, t)=\beta_{20}^{ \pm}(1, t)=\beta_{21}^{ \pm}(1, t)=0 .
$$

Therefore, here we find the usual result, namely, that there are no fixed poles at $J=1$.

At the point $J=0$, however, the situation is different. $J=0$ is an s-s point for $\phi_{00}$, an ordinary n-n point for $\phi_{11^{ \pm}}$and $\phi_{22}{ }^{ \pm}$, and an n-s point at the edge of the SN interval for $\phi_{10}$. For these amplitudes

$$
\beta_{\lambda \mu}\left(0, t_{-}\right)=\beta_{\lambda \mu}\left(0, t_{+}\right)^{*} .
$$

For the amplitudes $\phi_{20}$ and $\phi_{21} \pm, J=0$ is an n-s and n-n point, respectively, but the point lies within the SN interval. For these amplitudes there is the distinction between $J_{0_{+}}$and $J_{0_{-}}$. The real-analyticity condition leads to

$$
\beta_{2 \mu}^{ \pm}\left(0_{+}, t_{-}\right)=\beta_{2 \mu} \pm\left(0_{-}, t_{+}\right)^{*}, \quad \mu=0,1
$$

and neither quantity is necessarily equal to $\beta_{2 \mu} \pm\left(0_{+}, t_{+}\right)^{*}$ $=\beta_{2 \mu} \pm\left(0_{-}, t_{-}\right)$. Now, when we examine the unitarity condition near $J=0$ for an amplitude with an n-n

\footnotetext{
${ }^{14} \mathrm{It}$ is to be understood that only amplitudes with the same parity contribute in the unitarity condition; thus, for one parity the term involving $\beta_{20}$ is absent.
}

point, for example, we find

$$
\begin{aligned}
J^{-1}\left[\beta_{22} \pm(0, t)\right. & \left.-\beta_{22} \pm(0, t)^{*}\right]=2 i J^{-2}\left[\left|\beta_{22} \pm(0, t)\right|^{2}\right. \\
& \left.+\beta_{21} \pm\left(0_{+}, t\right) \beta_{21} \pm\left(0_{-}, t\right)^{*}\right]+ \text { terms of order } J^{-1}
\end{aligned}
$$

As before, the coefficient of $J^{-2}$ must be zero, but this no longer requires that $\beta_{22}{ }^{ \pm}(0, t)$ or $\beta_{21}{ }^{ \pm}\left(0_{ \pm}, t\right)$ individually vanish ${ }^{15}$; however, note that if one of these residues is different from zero, then so is the other.

Thus $\phi_{21}^{ \pm}$and $\phi_{22}^{ \pm}$can each have an n-n fixed pole at $J=0$. A similar analysis of the other unitarity conditions shows that $\phi_{10}$ and $\phi_{20}$ can each have an n-s fixed pole at the same point. Clearly, the essential requirement for a fixed pole at a nonsense point $J_{0}$ in one amplitude is that $J_{0}$ be interior to the SN interval for some amplitude with the same quantum numbers.

The necessary conditions imposed by unitarity, from which one can determine if fixed poles are allowed at an arbitrary nonsense point $J_{0}$, are given by

$$
\begin{aligned}
& \sum_{\nu} \beta_{\lambda \nu}{ }^{\mathrm{n} a}\left(J_{0_{+}}, t\right) \beta_{\nu \mu}^{\mathrm{n} b}\left(J_{0_{-}}, t\right)^{*}=0, \\
& \frac{\left[\beta_{\lambda \mu}^{a b}\left(J_{0_{+}}, t\right)-\beta_{\lambda \mu}^{a b}\left(J_{0_{-}}, t\right)^{*}\right]}{2 i}=\sum_{\nu} \beta_{\lambda \nu}{ }^{a \mathrm{~s}}\left(J_{0_{+}}, t\right) \beta_{\nu \mu}{ }^{b \mathrm{~s}}\left(J_{0_{-}}, t\right)^{*} \\
& +\sum_{\nu}\left[\beta_{\lambda \nu}{ }^{\mathrm{n} a}\left(J_{0_{+},}, t\right) \gamma_{\nu \mu}{ }^{\mathrm{n}}{ }^{b}\left(J_{0_{-},}, t\right)^{*}\right. \\
& \left.+\gamma_{\lambda \nu}{ }^{\mathrm{n} a}\left(J_{0_{+}}, t\right) \beta_{\nu \mu}{ }^{\mathrm{n} b}\left(J_{0_{-}}, t\right)^{*}\right],
\end{aligned}
$$

where $(a, b)$ can be either $(\mathrm{n}, \mathrm{n}),(\mathrm{n}, \mathrm{s})$, or $(\mathrm{s}, \mathrm{s})$. The sum in Eq. (8) is over nonsense while the first sum in Eq. (9) is over sense. Because of the presence in Eq. (9) of the quantities $\gamma_{\nu \mu}{ }^{n b}$, which do not appear in the Sommerfield-Watson transformed scattering amplitude, this equation provides no effective restriction on the $t$-channel discontinuities. Indeed, the same arguments which allow Eq. (8) to be satisfied nontrivially also allow the right-hand side of Eq. (9) to vanish. Thus, the surviving residues need not have cuts in $t$ nor need they depend on $t$ at all. This possibility is also not in conflict with Eq. (8). That the residues can be analytic in, or independent of, $t$ has important consequences which will be considered further in Sec. IV.

\section{Conditions for Fixed Poles}

The above results can easily be generalized to the case of several communicating channels. The main points will be stated without proof but the proofs follow directly from Eq. (8), where it is to be understood that one must consider the residues $\beta_{\lambda \mu}$ for all of the amplitudes in question and where the sum extends over all of the states which can contribute to the generalized unitarity condition at a given value of $t$. The results are

15 This point may be seen more clearly if we make explicit the singularities of $\phi_{21}^{ \pm}$on the SN interval. We have $\phi_{21} P(J, t)$ $=\overline{\boldsymbol{\beta}}_{21} P(J, t)[(J-1)(J+2)]^{-1 / 2}[J(J+1)]^{-1}$, where $\bar{\beta}^{P}$ is realanalytic in $J$ and $t$ with no cut in $J$. As $J$ approaches zero, we have $\phi_{21}^{P}(J \rightarrow 0, t) \rightarrow \mp(i / \sqrt{2}) J^{-1} \bar{\beta}_{21} P(0, t)$. We can make the identification $\beta_{21}{ }^{P}\left(0_{ \pm}, t\right)=\mp(i / \sqrt{2}) \bar{\beta}_{21} P(0, t)$, so that the coefficient of $J^{-2}$ is now given by $\left|\beta_{22} P(0, t)\right|^{2}-\frac{1}{2}\left|\beta_{21} P(0, t)\right|^{2}$. 
as follows:

Condition $I$. Let $t_{12}$ denote the threshold for a twobody state $1+2$. Then the continued partial-wave helicity amplitudes for the $t$-channel reaction $1+2 \rightarrow 3$ +4 can have fixed poles at the nonsense point $J_{0}$ if there is a communicating state $5+6$ such that $t_{56} \leq t_{12}$ $\leq t_{34}$ and $J_{0}$ is interior to the largest $\mathrm{SN}$ interval for the amplitudes of the reaction $5+6 \rightarrow 5+6$.

The allowed poles have the following properties:

(Ia) The "poles" may occur in the amplitudes for which $J_{0}$ is an n-s point or in those for which $J_{0}$ is an $\mathrm{n}-\mathrm{n}$ point, or simultaneously in both sets of amplitudes.

(Ib) The poles of each type, n-s or n-n, must occur in at least two amplitudes if they are to occur at all; the point $J_{0}$ is interior to the $\mathrm{SN}$ interval of one amplitude and exterior to the $\mathrm{SN}$ interval of the other amplitude.

(Ic) The residues of the allowed fixed poles need not, but may, have dynamical branch points at $t_{12}, t_{34}$, or $t_{56}$.

Condition I is sufficient to ensure at least the existence of the two amplitudes of property (Ib) for each case, n-s or n-n. State $5+6$ may coincide with $1+2$ or with both $1+2$ and $3+4$. Property (Ic) follows from the discussion following Eq. (9). If the spins of the two particles in the state $5+6$ are $\sigma_{5}$ and $\sigma_{6}$, then the "largest SN interval" referred to is $\left[\left(\sigma_{5}+\sigma_{6}-1\right)\right.$, $\left.-\left(\sigma_{5}+\sigma_{6}\right)\right]$.

One often encounters states with more than two particles in the unitarity sum. In the partial-wave decomposition of a three-body state, for example, one first couples the angular momentum of two of the particles, say, 2 and 3. The effective spin $\sigma_{23}$ of the pair $(2,3)$ can take on arbitrarily high values because of the relative orbital angular momentum in the c.m. system of the two particles. For given "spin" $\sigma_{23}$, the largest $\mathrm{SN}$ interval for the amplitudes in the reaction $1+(23) \rightarrow$ $1+(23)$ is $\left[\left(\sigma_{1}+\sigma_{23}-1\right),-\left(\sigma_{1}+\sigma_{23}\right)\right]$, and the threshold for each such amplitude is just the three-body threshold. The partial-wave projection of the amplitudes for any reaction leading to a three-particle state is, in effect, an infinite sum of states with increasing total spin. It follows that the "largest SN interval" referred to in condition $\mathrm{I}$ is the entire real $J$ axis whenever the reaction involves a state of three or more particles.

Condition I derives solely from the restrictions, or lack of them, in Eq. (8). In the case of coupled channels there is a source of fixed poles of a somewhat different nature. In this case Eq. (9) plays the important role. The new condition, which is complementary to $I$, is given by the following:

Condition II. The continued amplitudes for $1+2 \rightarrow$ $3+4$ can have fixed poles of the n-s type at $J_{0}$ if there are states $5+6$ and $7+8$ such that $t_{56} \leq t_{12}<t_{7} \leq t_{34}$ and $J_{0}$ is interior to the largest SN interval for $7+8 \rightarrow 7+8$, but exterior to that for $5+6 \rightarrow 5+6$. State $5+6(7+8)$ may coincide with $1+2(3+4)$.
In this case, n-n poles generally are prohibited at $J_{0}$ in the amplitudes for $1+2 \rightarrow 3+4$ by Eq. (8); an exception to this is discussed below in conjunction with condition III. In light of condition I, however, the presence of state $7+8$ permits $n-n$ fixed poles at $J_{0}$ in the amplitudes $3+4 \rightarrow 3+4$. It is the unitarity condition for the discontinuity in these amplitudes which determines whether poles are allowed in $1+2 \rightarrow 3+4$. In the present case the n-s poles are allowed via a relationship between the discontinuity on the lefthand side and the first term on the right-hand side of Eq. (9). One finds that the poles have the following property:

(IIa) The residue of a fixed pole in $1+2 \rightarrow 3+4$ allowed by II and some portion of the residue of the corresponding $n-n$ pole in $3+4 \rightarrow 3+4$ must have a right-hand cut in $t$ commencing at $t_{12}$.

It should be emphasized that it is not the entire residue of the $n-n$ pole in $3+4 \rightarrow 3+4$ that must have a cut in $t$. There may be several contributions, each of a different dynamical origin, to the $n-n$ pole allowed by condition I. One of these contri sutions may have the same dynamical origin as the n-s pole allowed in II; presumably it is this contribution that has the cut.

The above conclusions require modification at $n-s$ or n-n points of wrong signature. At wrong-signature nonsense points, the discontinuity across the left-hand cut of the Froissart-Gribov projection has poles, the so-called Gribov-Pomeranchuk poles, arising from the third double-spectral function. ${ }^{5}$ In order that these poles in the discontinuity not lead to essential singularities in the amplitude, there must be cuts in the $t$ plane which move with $J$. These cuts also arise from the third double-spectral function. ${ }^{2}$ As $J$ approaches a wrong-signature nonsense point, a cut moves up from the unphysical sheet in the $t$ plane to cover the righthand unitarity cut. ${ }^{16}$ The fixed pole in the discontinuity across the left-hand cut is now allowed to be a fixed pole in the amplitude.

The presence of the moving cut in the wrong-signature case means that the requirement that the point be interior to one of the $\mathrm{SN}$ intervals referred to in condition I can be relaxed. It should be pointed out, however, that the fixed poles which survive at wrong-signature nonsense points need not be related to the GribovPomeranchuk pole, not to the third double-spectral function. We now have, in addition to I and II, the following.

Condition III. At any wrong-signature n-s or n-n point, the continued amplitude can have fixed poles.

(IIIa) The residues of the poles need not have dynamical branch points in $t$.

To this list of conditions for fixed poles must be added one further restriction. The conditions which

${ }^{16}$ R. Oehme, Phys. Rev. Letters 18, 1222 (1967). 
have been given derive solely from $t$-channel unitarity. The allowed poles, however, are also subject to the restrictions of $s$-channel unitarity as embodied in the Froissart bound. Thus, there can be no fixed poles to the right of $J=1$.

It would appear that fixed poles are, at least in principle, a complication of spin. As we will see shortly, the fixed poles of interest in hadronic reactions are, in practice, a consequence of the existence of many-body states.

\section{LOCATION OF FIXED POLES}

Having established the set of conditions under which fixed poles are allowed, it is now necessary to show that the set is not empty for the interesting reactions. Only reactions with even baryon numbers will be considered.

\section{A. Hadronic Reactions Involving Fixed Poles}

The least-massive physical state involving two hadrons is the two-pion state. Since the pions have spin zero, there is no SN interval. Consequently, there are no fixed poles at right-signature points in the $\pi \pi$ elastic scattering amplitudes. The three amplitudes involved have zero baryon number and hypercharge, with isotopic spin, $G$ parity, signature, and parity given by $I^{G S P}=0^{+++}, 2^{+++}$, and $1^{+-}$. In the first two of these amplitudes, the wrong-signature fixed poles can be at $J_{0}=-1,-3, \ldots$, whereas for the last amplitude they can occur at $J_{0}=-2,-4, \ldots$.

Amplitudes for reactions leading to the two-pion state cannot satisfy condition I. In order for such amplitudes to satisfy condition II, they must have an $\mathrm{SN}$ interval. Thus only wrong-signature fixed poles can occur in $\pi \pi \rightarrow \eta \eta$ with $I^{G S P}=0^{+++}$, and in $\pi \pi \rightarrow K \bar{K}$ for $I^{G S P}=0^{+++}$and $1^{+\cdots}$.

An extremely important case is $\pi \pi \rightarrow N \bar{N}$. Here there are two helicity amplitudes $\phi_{00}$ and $\phi_{10}$, each of which can have $I^{G S P}=0^{+++}$or $1^{+--} . \phi_{00}$ has no SN interval, so only wrong-signature fixed poles are allowed. $\phi_{10}$ has the $\mathrm{SN}$ interval $[0,-1]$; condition II can be satisfied because the $4 \pi$ state, with its infinite SN interval, lies below the $N \bar{N}$ state. Thus $\phi_{10}$ can have right-signature n-s fixed poles for $\mathrm{I}^{G S P}=0^{+++}$at $J_{0}=0$, and for $I^{G S P}=I^{+-}$at $J_{0}=-1$. The residues for these fixed poles must have right-hand cuts. Of course, $\phi_{10}$ can also have the usual wrong-signature fixed poles for $I^{G S P}=0^{+++}$at $-1,-3, \ldots$ and for $I^{G S P}=1^{+--}$at $0,-2,-4, \ldots$.

Dolen, Horn, and Schmid, ${ }^{17}$ using finite-energy sum rules (FESR), have examined the amplitude $\phi_{10}$ with $I^{G S P}=1^{+-\infty}$ (the kinematic-singularity-free counterpart of which is usually called $B^{(-)}$) and have found the wrong-signature n-s pole at $J_{0}=0$. As we have seen, such a wrong-signature pole ${ }^{18}$ can have a part related

${ }^{17}$ R. Dolen, D. Horn, and C. Schmid, Phys. Rev. 166, 1768 (1968).

${ }_{18}$ It is true that condition II is also satisfied for this wrong- to the Gribov-Pomeranchuk singularity, which one hopes is small, and a part whose residue need not have a $t$-channel branch cut. It is noteworthy that the pole found by Dolen et al. ${ }^{17}$ appears to be dominated by the Born term.

Aviv and Horn, ${ }^{19}$ also using FESR, have examined the same amplitude at different points. They have found strong evidence for a pole at the right-signature point $J_{0}=-1$ and much weaker evidence for similar poles at -3 and $-5 .{ }^{19}$ These authors assert, in particular, that the pole at -1 is not dominated by the Born term but seems to be a cumulative background effect. This feature is consistent with the property of the condition which allows this pole, namely, the requirement that its residue has a right-hand branch point at the $\pi \pi$ threshold. We cannot explain the poles at $J_{0}=-3$ and -5 ; if they are really there, a new condition for their existence must be found.

The analysis of reactions such as $\pi \pi \rightarrow \pi V$ (A), where $V(A)$ is one of the established $G=-1$ vector (axialvector) mesons, is similar to that for $\pi \pi \rightarrow N \bar{N}$. In general, an amplitude for a reaction leading to a $\pi \pi$ state can have only those fixed poles allowed by conditions II and III.

The role played by the $\pi \pi$ state in limiting the fixed poles in the amplitudes considered above is played by the states $\pi K$ and $K K$ for $B=0$ amplitudes with $Y=1$, $I^{S P}=\frac{1}{2} \pm \pm$ or $\frac{3}{2} \pm \pm$ and $Y=2, I^{S P}=0^{++}$or $1^{++}$, respectively. A similar role is played by two baryon states of various hypercharge and isospin. For reactions leading to these states, only conditions II and III can be operative.

When other reactions are considered, one finds a proliferation of fixed poles. In all amplitudes having quantum numbers not yet mentioned, the lowest threshold involves a state with three or more pions. The largest SN interval for reactions leading to these states is $[-\infty, \infty]$, i.e., the entire real $J$ axis. Amplitudes with these quantum numbers always satisfy condition I and so can have fixed poles at any n-n or $\mathrm{n}$-s points. The same is true even in amplitudes with the two-body quantum numbers mentioned, if neither the initial nor final state in the reaction is the two-body state itself ; e.g., $\eta \eta \rightarrow \eta \eta, K \bar{K} \rightarrow K \bar{K}, N \bar{N} \rightarrow N \bar{N}$, etc., can have any n-n or $n-s$ fixed poles.

\section{B. Photoproduction : Fixed Poles in "Weak" Amplitude?}

So far our attention has been on purely hadronic reactions. Now we turn to the widely studied reaction $\gamma \pi \rightarrow N \bar{N}$. There are four helicity amplitudes in this

signature n-s pole. Property IIa need not hold, however, because of the presence of the moving cut.

${ }_{19}$ R. Aviv and D. Horn, Phys. Rev. Letters 21, 704 (1968). These authors assert that these poles are not in conflict with unitarity because of the ghost-eliminating factor. We have seen, however, that even if the poles are factored out of the amplitude, they are still present in the unitarity condition and must be explained by a mechanism which is consistent with that condition 
case : $\phi_{10}{ }^{+}$and $\phi_{11}{ }^{+}$with $I^{G S P}=0^{-\cdots}, 1^{+--}$, or $1^{-++}$; $\phi_{10}^{-}$with $I^{G S P}=0^{--+}, 1^{+-}$, or $1^{-+}$; and finally, $\phi_{11^{-}}$with $I^{G S P}=0^{-+-}, 1^{++-}$, or $1^{--+}$. The amplitudes $\phi_{11}^{ \pm}$also contain secondary contributions with the $I, G$, and $P$ noted, but with opposite signature. Photoproduction of charged pions involves the amplitudes with $I^{G}=1^{+}$and $1^{-}$and neutral pions those with $I^{G}=1^{+}$and $0^{-}$. The two-pion state corresponds to the lowest branch point in $\phi_{10}{ }^{+}$and $\phi_{11}{ }^{+}$with $I^{G}=1^{+--}$. In all of the other amplitudes, the three-pion state is lowest. All n-n or n-s fixed poles are allowed because the lowest threshold state is neither the initial nor the final state.

At this point let us digress to refute the old argument for fixed poles in photoproduction. It has been suggested that fixed poles can occur at nonsense points in weak amplitudes. The argument states that when (electromagnetic or) "weak" interactions of hadrons are computed to lowest order in the weak coupling, then exact bilinear unitarity, which ordinarily would forbid fixed poles, does not apply. If the computation were carried out to all orders in the weak coupling, then the fixed pole allowed by linear unitarity would be transformed into a moving pole in the usual way. ${ }^{20}$

This argument would lead to a curious result, however, if it were valid for photoproduction. The weak trajectory in this case would have the form $\alpha(t)=J_{0}$ $+e \epsilon(t)$, where we have made explicit the presumption that the pole is fixed at $J_{0}$ to lowest order in the charge $e$. The pole term, correct to all orders in $e$, would have the form

$$
\beta_{\gamma \pi, N \bar{N}} /\left[J-\left(J_{0}+e \epsilon(t)\right)\right],
$$

where, because exact unitarity is valid, the residue factorizes:

$$
\beta_{\gamma \pi, N \bar{N}}=\gamma_{\gamma \pi} \gamma_{N \bar{N}}
$$

Because the pole is coupled to an external photon line, the residue factor $\gamma_{\gamma \pi}$ must be of order $e$, at least. There are two possibilities for the $\gamma_{N \bar{N}}$ factor:

(i) $\gamma_{N \bar{N}} \sim e^{2}$, at least. In this case, the pole term should be neglected if one retains only terms of lowest order in $e$.

(ii) $\gamma_{N \bar{N}} \sim e^{0}=1$.

In the latter case the pole would also be present in the $N \bar{N}$ scattering amplitude with full residue $\gamma_{N} \bar{N}^{2} \sim e^{0}$ $=1$. Thus we are faced with the curious circumstance of a "weak" pole which contributes strongly to a hadronic process. Furthermore, the pole does not go away when one neglects all but the lowest order in $e$; the pole merely becomes fixed at $J=J_{0}$. Since it is untenable to have a strong contribution of a weak pole, possibility (ii) must be rejected.

${ }^{20}$ R. Dashen and S. Y. Lee, Phys. Rev. Letters 22, 366 (1969); J. B. Bronzan, I. S. Gerstein, B. W. Lee, and F. E. Low, ibid. 18, 32 (1967); V. Singh, ibid. 18, 36 (1967).
As it turns out, it is possible to include terms of higher order in $e$ and retain all of the poles allowed so far. Isospin and $G$ parity are no longer useful in classifying the states but one can look at the different charge states. In the case of charged pions, the threshold for the $\gamma \pi$ state is coincident with the thresholds for states with two or more photons and a pion (as well as with the position of the pion pole). ${ }^{21}$ Again the infinite SN interval associated with these many-body states now allows all $n-s$ and $n-n$ poles. A similar result holds in the neutral pion case where the lowest two-body threshold for the $\gamma \gamma$ state coincides with that for three or more photons (and with the position of the photon pole).

\section{NATURE OF FIXED POLES}

Now that we know where fixed poles are allowed, we must next look for dynamical mechanisms which can actually generate them. One clue to their origin is that they are fixed at points which are nonsensical in the $t$ channel. This fact suggests that the fixed poles need have little to do with particles or resonances, i.e., with ordinary Regge trajectories, in the $t$ channel.

\section{A. Possible Mechanisms for Fixed Poles}

Poles which are allowed by condition III will not contribute to the asymptotic behavior of the physical amplitude, because of the wrong-signature factor. This is true of the pole itself even if it were to occur in the residue of some moving pole; in this case, however, the behavior of the residue at the position of the pole is altered according to the now-familiar "new dip mechanism."2 Clearly, the Gribov-Pomeranchuk pole is of this type, but what possibly has not been fully appreciated is that fixed poles arising from sources other than the third double-spectral functions are allowed as well. The fixed pole found by Dolen, Horn, and Schmid, ${ }^{17}$ for example, appears to arise mainly from the Born term in the $\pi N$ scattering amplitude.

The general form of the contribution of such $s$ - and $u$-channel pole terms to the absorptive part of a singularity-free physical amplitude is

$$
\begin{aligned}
\left(\pi / 2 q_{12} q_{34}\right)\left[\Gamma_{s} \delta\left(z_{s}-z\right)\right. & \left.+\Gamma_{u} \delta\left(z_{u}+z\right)\right] \\
& \equiv \pi\left[\Gamma_{s} \delta\left(s-M_{s}^{2}\right)+\Gamma_{u} \delta\left(u-M_{u}{ }^{2}\right)\right] .
\end{aligned}
$$

For the continued amplitudes of definite signature, the contribution is

$$
\left(1 / 2 q_{12} q_{34}\right)\left[\Gamma_{s} \xi_{\lambda \mu}(z) e^{J}(z)+S(-1)^{\lambda} \boldsymbol{\Gamma}_{u} \xi_{\lambda,-\mu}(z) e_{\lambda,-\mu}^{J}(z)\right] .
$$

It does not matter to the signatured amplitudes, with which we have been dealing, if the point $J_{0}$ where the fixed pole is located is a right- or wrong-signature point

${ }^{21}$ Care must be exercised in applying condition $I$ to states involving photons. The transverseness of the photon means that certain helicity states are forbidden. 
in the physical amplitude. Thus, contributions arising from Born terms may survive even at right-signature points, if they are allowed by condition I. A similar comment applies to the single-spectral functions which generally accompany the stable-particle poles. Born-like contributions cannot arise under condition II because they do not have the requisite right-hand cut in $t$.

Clearly, these Born contributions have characteristics peculiar to the $s$ and $u$ channels. It is possible that other contributions characteristic of these channels can exist; for example, one might expect that $s(u)$ channel effects can arise from the double-spectral functions $\rho_{s t}\left(\rho_{u t}\right)$ and $\rho_{s u}$. For lack of a better term, we will refer to these contributions as "residual crossedchannel effects."

Models have already been constructed which attempt to include Regge poles and the effects of other channels. The prime example of this type is the Regge-plusresonance, or interference model. ${ }^{22}$ Objections to this model were raised and quickly given firm support when it was shown, through the use of FESR, that the model committed double counting. ${ }^{17}$ The main implications of the FESR have been formulated into the principle of Dolen-Horn-Schmid duality, which requires that resonances (and background) in the $s$ or $u$ channels sum up to give the $t$-channel Regge amplitudes. It might be thought that the concept of residual crossedchannel effects introduced above is in conflict with duality. This is not the case, however, because these effects have equally valid descriptions in the $s$ or $u$ channel, whence they arise, and in the $t$ channel, as fixed poles.

Finally, we mention one other possible mechanism for fixed poles. Certain models for coupling Regge poles to external particles with spin exhibit fixed poles at rightor wrong-signature points. ${ }^{23}$ These models are based on the technique of summing certain classes of Feynman diagrams, usually of the type associated with $\rho_{s t}$ and $\rho_{u t}$. The fixed poles in question occur in the residues of moving poles at $n$-s points. It is not yet certain that these particular poles survive when other classes of diagrams are considered. If they do survive, however, they fall in the category of, and provide a possible mechanism for, poles allowed by condition II.

\section{B. Channel Characteristics}

We have seen that $t$-channel fixed poles can reflect behavior purely characteristic of the $s$ or $u$ channels, i.e., the residual crossed-channel effects. It is of interest to determine, therefore, to what extent one can classify the various contributions to the Regge asymptotic series according to channel. In this section we will try to give a firmer meaning to this admittedly imprecise terminology. The Regge description, however, is not yet a dynamical theory; to attain the stated goal, it

${ }^{22}$ V. Barger and D. Cline, Phys. Rev. 155, 1792 (1967).

${ }^{23}$ A. Swift, Phys. Rev. 166, 1621 (1968).

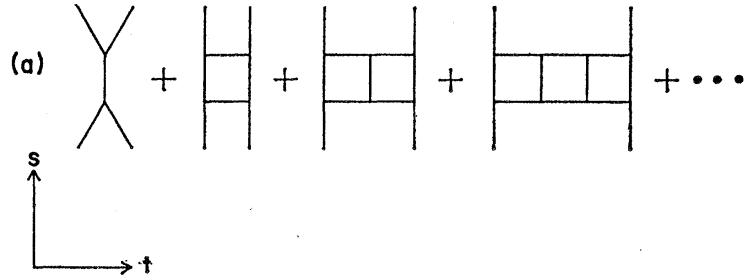

(b)

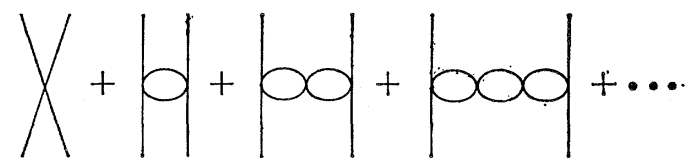

FIG. 2. (a) Feynman diagrams in a $t$-channel ladder sum. (b) Reduced ladder diagrams which survive in the limit of large $s$ and which lead to Regge behavior.

will be necessary to resort to some common models. Thus, the interpretation given here is subject to change.

A contribution to the scattering amplitude will be regarded as characteristic of a given channel if it corresponds to the exchange of one or more particles in that channel. Thus a single-particle pole in an amplitude is characteristic of the channel in which it occurs; a box diagram having two-particle discontinuities in two channels is characteristic of both.

What is the situation for a Regge trajectory? From the point of view of the van Hove model, ${ }^{24}$ for example, a $t$-channel trajectory corresponds to an infinite sum of particle exchanges in the $t$ channel; one particle at each physical value of $J$ (i.e., at sense points of right signature). With the definition given above, a van Hove-like trajectory is certainly characteristic of the $t$ channel alone.

Trajectories can be generated in field theory by summing ladder diagrams [see Fig. 2(a)]. With the above interpretation, it appears that all terms in the sum are characteristic of the $s$ channel and all but the first are characteristic of the $t$ channel. In order to obtain the trajectory, however, it is necessary to go to the limit of large $s$ and to retain only the leading term. In this limit we are left with terms which correspond to those of Fig. 2(b), where the particle lines are associated with two-(dimensional) momenta. Clearly, all $s$-channel characteristics have disappeared; so in this model also, the trajectory is characteristic of the $t$ channel alone.

The models considered above are consistent with the well-known property that Regge trajectories only have right-hand ( $t$-channel) dynamical branch points.

We are thus led to formulate these results, and those of Sec. IV A, as a basic principle: Regge trajectories are characteristic only of the channel in which they occur, but fixed poles in a channel can be characteristic of the crossed channels. We now consider the most important application of this principle.

${ }^{24}$ L. van Hove, Phys. Rev. Letters 24B, 183 (1967). 


\section{SOME CONSEQUENCES OF EXISTENCE OF FIXED POLES}

The existence of fixed poles would appear to be a complication for Regge theory. Here we will show, instead, that the fixed poles may simplify the theory considerably.

\section{A. Conspiracy as Fixed Poles}

When all kinematic simularities are removed from $t$-channel helicity amplitudes, certain constraint relations exist at $t=0$ among amplitudes with different quantum numbers. Constraints also exist between amplitudes with the same quantum numbers at thresholds and pseudothresholds, but these are well understood in terms of the threshold behavior of states with low orbital angular momentum. The various ways in which the constraints may be satisfied have been classified as follows. ${ }^{25}$

(1) Evasion: Constraints are satisfied by relations among the residues of different poles, but the trajectories themselves are unrelated at $t=0$.

(2) Daughters: Constraints are satisfied by infinite sequences of trajectories with the same quantum numbers, the daughters lying lower than the parent trajectory and spaced by two units of angular momentum at $t=0$.

(3) Conspiracy: Constraints are satisfied by relationships between trajectories with different quantum numbers at $t=0$.

There are basically two types of conspiracies. The first type, which may be called daughter-like conspiracy, requires the existence of odd-numbered daughters, spaced by two units of angular momentum at $t=0$ and all having the same quantum numbers. The odd daughters, however, have signature and parity opposite to that of the parent and the leading odd daughter lies one unit below the parent at $t=0$. In this case, terms with the same $s$ dependence occur in different amplitudes and the constraints can be satisfied in a nontrivial fashion; the terms with the same $s$ dependence can be identified as a "minor" contribution ${ }^{26}$ of the parent or even daughter in one amplitude and as the odddaughter contribution in the other amplitude. To the extent that one can understand this existence of daughters as a requirement of analyticity at $t=0$, then this type of conspiracy, wherein all of the trajectories involved are related to the same parent, is perfectly natural.

The second type of conspiracy involves two-parent trajectories and their daughter sequences. The parent trajectories have the same signature, but opposite parity, and they coincide at $t=0$. This type of behavior, which requires the coincidence of two different parents, may be called true conspiracy.

${ }^{25}$ E. Leader, Phys. Rev. 166, 1599 (1968).

${ }^{26}$ L. Jones and H. K. Shepard, Phys. Rev. 175, 2117 (1968).
Consider now a Regge trajectory $\alpha$ which can be exchanged in the $t$ channel of some reaction. At the value $t_{i}$, where $\operatorname{Re} \alpha(t)$ passes through a right-signature point $J_{i}$ in the sense region, there should be a particle of spin $J_{i}$ and $(\text { mass })^{2}=t_{i}$ [one can expect to see the particle if $\operatorname{Im} \alpha\left(t_{i}\right)$ is not too large]. One can use Feynman techniques to compute the general oneparticle-exchange contributions to the various $t$-channel helicity amplitudes for each particle on the trajectory. ${ }^{27}$ An interesting pattern emerges, namely, the behavior of this entire set of contributions to the various helicity amplitudes at $t=0$ is consistent with evasion, or daughters, or the daughter-like conspiracy. One never finds behavior consistent with true conspiracy from exchanges characteristic of the $t$ channel. ${ }^{28}$

The classic example of the general property occurs in the reactions $\gamma p \rightarrow \pi^{+} n$ and $n p \rightarrow p n$, where the sharp foward peaking had led some authors ${ }^{29}$ to invoke a conspiring pion. The contribution of a conspiring pion trajectory to the kinematic-singularity-free amplitudes for these reactions is finite at $t=0$. Ordinary onepion exchange, however, gives a contribution which vanishes at $t=0$. The same is true for the one-particle exchange of any other "particle" on the pion or conspirator trajectories. ${ }^{30}$

That one-particle exchanges can never enter into true conspiracy is easy to understand. Opposite-parity exchanges are inherently independent and so they must satisfy the constraints separately, i.e., evasively. It is true that one-particle exchanges with high spin are not consistent with Regge behavior. Their properties are not irrelevant, however, because a suitable generalization of the van Hove model to the case of spin has been made. ${ }^{28}$ The model exhibits all of the requisite Regge properties except, of course, that of true conspiracy.

There is one other interesting feature of one-particleexchange calculations that is relevant in this context. Any $s$ - or $u$-channel exchange, when expressed in terms of $t$-channel helicity amplitudes, automatically satisfies the constraint conditions in a manner consistent with true conspiracy. A similar comment applies to any contribution, such as a box diagram, which retains some of the character of the $s$ or $u$ channels.

Since fixed poles, in contrast to ordinary trajectories, can reflect these residual crossed-channel effects, it is only natural that the fixed poles be accused of conspiracy. Indeed, this is necessary if our standard ideas concerning the $t=0$ coupling of external particles to those exchanged internally are not to be drastically

27 B. Diu and M. Le Bellac, Nuovo Cimento 53 A, 158 (1968); G. Cohen-Tannoudji, Ph. Salin, and A. Morel, CERN Report No. TH 860, 1967 (unpublished); L. Durand, III, Phys. Rev. 154, 1537 (1967).

${ }^{28}$ H. F. Jones and M. D. Scadron, Phys. Rev. 171, 1809 (1968).

${ }^{29} \mathrm{~F}$. Arbab and J. W. Dash, Phys. Rev. 163, 1603 (1967); R. J. N. Phillips, Nucl. Phys. B2, 394 (1967) ; J. S. Ball, W. R. Frazer, and M. Jacob, Phys. Rev. Letters 20, 518 (1968).

${ }^{30}$ S. D. Drell and J. D. Sullivan, Phys. Rev. Letters 19, 268 (1967); S. Frautschi and L. Jones, Phys. Rev. 164, 1918 (1968). 
changed as the transition to Regge exchange is made. We are thus led to formulate another principle, namely, that only fixed poles enter into true conspiracy.

That fixed poles can conspire is a consequence of the conditions for their existence. First note, however, that although property (Ib) states that fixed poles must occur in at least two amplitudes, this is not the pair which contains the different conspirators. The reason is simply that these amplitudes, which appear in the same unitarity condition, must have the same quantum numbers, whereas the conspirators have opposite parity. The conditions which allow fixed poles, however, depend either on the size of some SN interval or on whether the nonsense point in question has the wrong signature. It is clear that if these conditions are met at a nonsense point in an amplitude of one parity, they generally are met as well for the corresponding point in the constrained opposite-parity amplitude. The fixed poles thus allowed can be made to satisfy the constraints at $t=0$ merely by a relation between their residues.

It should be noted that in the two cases where the conspiracy phenomenon is firmly established, namely, in photoproduction and $n-p$ charge-exchange scattering, condition I allows both of the $I^{G}=1^{-}$opposite-parity $t$-channel amplitudes involved in the conspiracy constraint $^{31}$ to have fixed poles.

In the context of group-theoretical predictions, assuming that the $S O(3,1)$ classifications are meaning$\mathrm{ful},{ }^{32}$ the foregoing implies that ordinary trajectories (and perhaps some fixed poles) should be identified with Toller poles of class I or II and only fixed poles should be identified with class III. Since the fixed poles occur at integral $J$, however, they need not have infinite daughter sequences. In the same context, but for $t<0$, it should be noted that the existence of fixed poles requires that the full richness of the spectrum of $S O(2,1)$ be utilized ${ }^{33}$; i.e., in addition to the principal series representations, which are ordinarily identified with the Regge background integral, one must use the discrete series representations, which can be identified with the nonsense points.

\section{B. Miscellany}

Here we mention briefly several disjoint topics wherein fixed poles can play an important role. The list is by no means meant to be exhaustive.

(1) Superconvergence: If, for some range of $t$, the leading trajectory lies below a nonsense point, the amplitude to which it contributes is either superconvergent or has a fixed pole at that point. Formerly, fixed poles were allowed only at wrong-signature points. Now the amplitude will not be superconvergent at right-signature points where it has a fixed pole.

${ }^{31}$ R. Diebold, Phys. Rev. Letters 22, 204 (1969) ; F. J. Gilman, Stanford Linear Accelerator Center Report No. TH 589, 1969 (unpublished).

${ }_{32}$ M. Levy-Leblond, Nuovo Cimento 45A, 772 (1966).

${ }^{33}$ J. F. Boyce, J. Math. Phys. 8, 675 (1967).
(2) Large-angle scattering: If ordinary trajectories are more or less linear in $t$, then for $s$ sufficiently large and $t$ fixed well outside the forward and backward peaks, all of $t$ - and $u$-channel trajectories will have fallen to very low values. If, further, there are fixed poles with residues weakly dependent on $t$ (or $u$ ), they will dominate the moving poles and become, so to speak, the bridge between the forward and backward peaks. In this region, however, third-double-spectral-function effects, such as Regge cuts, will be relatively more important than in the region of small $t$ or $u$.

(3) Dip filling: In a differential cross section, the dip which is expected at the value of $t$ where the leading trajectory passes through a nonsense point may be partially or completely filled if there is a fixed pole at that point. Thus, dip filling need not be associated with the behavior of the residue for that trajectory.

A case in point is the behavior of the residue of the $A_{2}$ pole near $\alpha=0$ in the $t$ channel of the reactions $\pi^{-} p \rightarrow \eta n$ and $K^{+} p \rightarrow K^{0} \Delta^{++}$. Note also that the apparent leveling off of the $A_{2}$ trajectory ${ }^{34}$ may be due to the emergence of the allowed fixed pole as the dominant contribution for $t \approx-1.0(\mathrm{BeV} / \mathrm{c})^{2}$.

(4) Clearly, old phenomenological analysis of reactions in which fixed poles are now expected to contribute should be redone.

\section{CONCLUSIONS}

We have shown that, under certain conditions, fixed $J$-plane poles are allowed by unitarity, even at rightsignature points. Only those poles which appear explicitly in the Froissart-Gribov projection have been considered, namely, those which arise at nonsense points from the $J$-plane singularities of the rotation functions of the second kind. The conditions for the existence of these poles have been determined and several mechanisms which may generate them have been considered.

Some of the less obvious distinctions between fixed poles and ordinary trajectories have also been considered. It has been shown that fixed poles can exhibit characteristics peculiar to the cross channels, whereas ordinary trajectories, at least those generated by any of the models currently in vogue, exhibit characteristics only of the channel in which the pole occurs. On the basis of these channel characteristics, we have seen that fixed poles provide the natural explanation of conspiracy in boson channels.

The existence of fixed poles revives the hope that Regge cuts are unimportant at high energies. Whether these poles do, in fact, account for all of the phenomena which currently are thought to necessitate cuts, must be verified separately in each case. The relatively simple properties of the poles, and their economical parametrization, are sufficient reasons for exploring this possibility.

${ }^{34}$ R. J. N. Phillips and W. Rarita, Phys. Letters 19, 598 (1965). 